\title{
On a composite functional equation fulfilled by modulus of an additive function
}

\section{TOMASZ KOCHANEK}

Dedicated to Janos Aczel on the occasion of his birthday.

Abstract. We deal with the problem of determining general solutions $f: \mathbb{R} \rightarrow \mathbb{R}$ of the following composite functional equation introduced by Fechner:

$$
f(f(x)-f(y))=f(x+y)+f(x-y)-f(x)-f(y) .
$$

Our result gives a partial answer to this problem under some assumptions upon $f(\mathbb{R})$. We are applying a theorem of Simon and Volkmann concerning a certain characterization of modulus of an additive function. A new proof of their result is also presented.

Mathematics Subject Classification (2000). 39B12.

Keywords. Composite functional equation, additive function.

\section{Introduction}

In this note we are dealing with the composite functional equation

$$
f(f(x)-f(y))=f(x+y)+f(x-y)-f(x)-f(y)
$$

introduced by Fechner [1] in connection with the elementary identity

$$
|| x|-| y||=|x+y|+|x-y|-|x|-|y|,
$$

which goes back to Tarski [5]. In his paper Fechner established all solutions $f: \mathbb{R} \rightarrow \mathbb{R}$ which are either continuous at zero or monotonic (see $[1$, Theorem 11] and [1, Theorem 13], respectively). One step to derive a general solution (with no regularity assumptions) has been done in his paper as well (see

This research has been supported by the scholarship from the UPGOW project co-financed by the European Social Fund. 
[1, Theorem 7]). Namely, in the case where $G$ is an Abelian group uniquely divisible by 2 , every solution $f: G \rightarrow G$ of $(*)$ with $f(G) \subset-f(G)$ has to be an idempotent additive self-mapping on $G$. Our goal is to provide a wider (but still partial) answer to the question about the general form of solutions of Eq. (*) in the case $G=\mathbb{R}$.

For any function $f: \mathbb{R} \rightarrow \mathbb{R}$ we define

$$
S=f(\mathbb{R}), \quad Z=S \cap(-S), \quad P=S \backslash Z .
$$

If $f$ is a solution of $(*)$ then, in view of [1, Lemma 1], $\left.f\right|_{S}$ is the identity function and

$$
f(n x)=n f(x) \quad \text { for all } x \in \mathbb{R}, n \in \mathbb{N} .
$$

It is also easily verified that $(S,+)$ forms a subsemigroup of the group $(\mathbb{R},+)$ and hence $(Z,+)$ is a subgroup of $(\mathbb{R},+)$. Moreover, $(P,+)$ is a semigroup. Indeed, if $t, u \in P$ then clearly $t+u \in S$ and $f(t+u)=t+u$. If $t+u \in Z$ then we would have $t+u=-v$ for some $v \in S$. Therefore, $-t=u+v \in S$ which yields $t \in Z$; a contradiction. Consequently, $t+u \in S \backslash Z=P$.

By virtue of (1.2), we have

$$
S=\mathbb{Q}^{+} \cdot S, \quad Z=\mathbb{Q}^{+} \cdot Z, \quad P=\mathbb{Q}^{+} \cdot P,
$$

where $\mathbb{Q}^{+}:=\mathbb{Q} \cap(0, \infty)$.

Note that the assumption of [1, Theorem 7] quoted above says that $S$ is a group, i.e. $P=\emptyset$. Our aim is to solve Eq. $(*)$ in the more general case where $S \cup(-S)$ forms a group (which is equivalent to $S \cup(-S)=S-S$ ). Because of Fechner's result, we may (and we actually do) assume that $P \neq \emptyset$.

Our main result reads as follows.

Theorem 1. Assume $f: \mathbb{R} \rightarrow \mathbb{R}$ is a function satisfying the following conditions:

$\left(\mathrm{H}_{1}\right) S \cup(-S)=S-S$,

$\left(\mathrm{H}_{2}\right)$ there exists $\xi \in P$ such that

$$
P \subset \bigcup_{n \in \mathbb{N}}\left(\frac{1}{n} \xi+P\right)
$$

and

$$
\bigcap_{n \in \mathbb{N}}(n \xi+P)=\emptyset \text {. }
$$

Then $f$ is a solution of Eq. (*) if and only if there exist: an additive function $A: \mathbb{R} \rightarrow \mathbb{R}$, a subgroup $R$ of $\mathbb{R}$ such that $\left.A\right|_{R}: R \rightarrow A(\mathbb{R})$ is an isomorphism, and a function $z: \mathbb{R} \rightarrow Z$, where $Z \subset \operatorname{ker} A$ is a group, with the properties:

(i) $\left.z\right|_{-D}$ is additive, where $D=A^{-1}([0, \infty))$,

(ii) $z(x)=-z(-x)+z\left(-\left.A\right|_{R} ^{-1}(|A(x)|)\right)$ for all $x \in D$,

(iii) $z(x)=x$ for all $x \in Z$, 
such that

$$
f(x)=\left.A\right|_{R} ^{-1}(|A(x)|)+z(x) \quad \text { for all } x \in \mathbb{R} .
$$

Conditions (i)-(iii) give a clear description of any function $z$ which comes into play. Namely, when we ensure that $z$ is additive on the semigroup $-D$ and $z$ is identity on $Z \subset \operatorname{ker} A \subset-D$, then the values of $z$ on the set $D$ are well-defined by formula (ii). As we see, there is a lot of freedom for the choice of function $z$; in a very particular case it may be an arbitrary additive function being identity on $Z$ and vanishing on $R$.

We also have a variety of additive functions $A$ (and subgroups $R$ ) which yield solutions of Eq. (*) with the aid of (1.6). In a particular case $A$ may be an arbitrary idempotent additive function (i.e. $A \circ A=A$ ) and $R=A(\mathbb{R})$. Then $\left.A\right|_{R}$ is simply the identity function on $R$. In such a case the term $\left.A\right|_{R} ^{-1}(|A(x)|)$ reduces to $|A(x)|$.

Remark 1. If $A$ and $z$ are as above, then the following conditions hold:

(iv) $\left.z\right|_{D}$ is additive,

(v) $z(x)=-z(-x)+z\left(-\left.A\right|_{R} ^{-1}(|A(x)|)\right)$ for all $x \in \mathbb{R}$,

(vi) $z(x)=0$ for all $x \in D \cap R$.

Proof. For (iv) note that in view of (ii) and (i), for $x, y \in D$ we have

$$
\begin{aligned}
z(x)+z(y) & =z\left(-\left.A\right|_{R} ^{-1}(|A(x)|)\right)+z\left(-\left.A\right|_{R} ^{-1}(|A(y)|)\right)-z(-x)-z(-y) \\
& =z\left(-\left.A\right|_{R} ^{-1}(A(x)+A(y))\right)-z(-x-y)=z(x+y) .
\end{aligned}
$$

To get (v) it is enough to fix $x \in-D$ and put $-x$ in the place of $x$ in formula (ii). Finally, for an arbitrary $x \in D \cap R$ we have $x=\left.A\right|_{R} ^{-1}(|A(y)|)$ for a certain $y \in \mathbb{R}$ and putting this into equation (ii) we get $z(x)=-z(-x)+z(-x)=0$.

Remark 2. If $\left(\mathrm{H}_{1}\right)$ holds, $P \neq \emptyset$ and $S$ is bounded from one side, then $\left(\mathrm{H}_{2}\right)$ holds.

Proof. If $S$ (and hence also $P$ ) is bounded from one side, then condition (1.4) is trivially fulfilled with an arbitrary $\xi \in P$. Now, we show that (1.5) also holds with any $\xi \in P$. Suppose, on the contrary, that there is $p \in P$ such that $p-\frac{1}{n} \xi \notin P$ for $n \in \mathbb{N}$. In view of (1.3) and $\left(\mathrm{H}_{1}\right)$, we have

$$
p-\frac{1}{n} \xi \in P-P \subset S-S=S \cup(-S) .
$$

Since $p-\frac{1}{n} \xi \notin P$, we have either $p-\frac{1}{n} \xi \notin S$, or $p-\frac{1}{n} \xi \in Z$. By (1.7), in both cases $p-\frac{1}{n} \xi \in-S$, which implies $\xi-n p \in S$ for all $n \in \mathbb{N}$ and contradicts the fact that $S$ is bounded from one side.

In the last section we will show that the situation where $\left(\mathrm{H}_{2}\right)$ is not valid is possible and produces new solutions of Eq. (*). They "pretend" to behave partially like an additive function and partially like the absolute value function. 


\section{The proof}

One of the crucial parts of the proof is a construction of an additive mapping which is positive on a given semigroup (and nowhere else). To this end we apply a method used by Sablik in the proof of [3, Proposition 3.1]. Our strategy is also based on a theorem of Simon and Volkmann [4] which says that a function $g: \mathbb{R} \rightarrow \mathbb{R}$ satisfies the equation

$$
g(x)+g(y)=\max \{g(x+y), g(x-y)\} \quad \text { for all } x, y \in \mathbb{R}
$$

if and only if there is an additive function $A: \mathbb{R} \rightarrow \mathbb{R}$ such that

$$
g(x)=|A(x)| \text { for all } x \in \mathbb{R} .
$$

A new proof of this statement, quite different from the original one, may be found in the next section.

Proof of Theorem 1 . Suppose $f: \mathbb{R} \rightarrow \mathbb{R}$ is a solution of $(*)$ which fulfils $\left(\mathrm{H}_{1}\right)$ and $\left(\mathrm{H}_{2}\right)$. Denote by $X$ the group $S \cup(-S)$ and by $\widetilde{X}$ the quotient group $X / Z$. Let also $\widetilde{S}=\{x+Z: x \in S\}$. Plainly, $\widetilde{S} \cup(-\widetilde{S})=\widetilde{X}$. Moreover, $\widetilde{S} \cap(-\widetilde{S})=\{0\}$ (0 stands here for the coset $Z$ ). Indeed, suppose $t \in S$ and $t+Z \in-\widetilde{S}$. Then there is $u \in S$ such that $t+Z=-u+Z$, i.e. $t+u \in Z$. Thus $-t-u \in S$ which gives $f(-t-u)=-t-u$ and $-t=f(-t-u)+u \in S$. Consequently, $t \in-S$ and therefore $t \in Z$, which yields $t+Z=Z$.

Let $\widetilde{S}^{*}=\widetilde{S} \backslash\{0\}$. Then, obviously, $\widetilde{S}^{*} \cup\left(-\widetilde{S}^{*}\right)=\widetilde{X} \backslash\{0\}$ and $\widetilde{S}^{*} \cap\left(-\widetilde{S}^{*}\right)=\emptyset$. Moreover, $\widetilde{S}^{*}+\widetilde{S}^{*} \subset \widetilde{S}^{*}$. Indeed, for every $t, u \in P$ we have $(t+Z)+(u+Z)=$ $(t+u)+Z \in \widetilde{S}$. If $(t+u)+Z=0$, then we would have $t+u \in Z$ and the argumentation used in the preceding paragraph shows that $t \in Z$, which is impossible.

Now, we are going to construct an additive function $a: \widetilde{X} \rightarrow \mathbb{R}$ such that

$$
a^{-1}((0, \infty))=\widetilde{S}^{*} .
$$

To this end pick any $\xi \in P$ satisfying conditions (1.4) and (1.5). For every $x \in P$ we define

$$
A_{x}=\left\{\frac{m}{n} \in \mathbb{Q}: m \in \mathbb{Z}, n \in \mathbb{N} \text { and } n x+m \xi \in P\right\} .
$$

By means of (1.4), there is $n \in \mathbb{N}$ such that $x \in \frac{1}{n} \xi+P$. Hence, in view of (1.3), we have $n x-\xi \in P$, which implies inf $A_{x}<0$. In what follows we show that inf $A_{x}>-\infty$.

Suppose $\frac{m}{n} \in A_{x}$ and $\frac{m^{\prime}}{n^{\prime}}>\frac{m}{n}$ for some $m, m^{\prime} \in \mathbb{Z}$ and $n, n^{\prime} \in \mathbb{N}$. Then $m^{\prime} n-m n^{\prime}>0$ and since $P$ is a semigroup, we obtain

$$
n\left(n^{\prime} x+m^{\prime} \xi\right)=n^{\prime}(n x+m \xi)+\left(m^{\prime} n-n^{\prime} m\right) \xi \in P .
$$


Thus, (1.3) implies $\frac{m^{\prime}}{n^{\prime}} \in A_{x}$. We have just shown the following condition:

$$
\text { if } q \in A_{x} \text { and } \mathbb{Q} \ni q^{\prime}>q \text {, then } q^{\prime} \in A_{x} \text {. }
$$

Consequently, to obtain $\inf A_{x}>-\infty$ it is enough to show that $-n \notin A_{x}$ for at least one $n \in \mathbb{N}$. This fact, however, follows from condition (1.5), since otherwise we would have $x-n \xi \in P$ for every $n \in P$, i.e. $x \in \bigcap_{n \in \mathbb{N}}(n \xi+P)$.

Let $x, y \in P$ and suppose $x+Z=y+Z$. Then $y=x+z$ for a certain $z \in Z$. Now, if $n x+m \xi \in P$ for some $m \in \mathbb{Z}$ and $n \in \mathbb{N}$, then

$$
n y+m \xi=(n x+m \xi)+n z \in P+Z=P,
$$

which proves $A_{x} \subset A_{y}$. Interchanging the roles of $x$ and $y$ we obtain $A_{x}=A_{y}$. Therefore, we may define a function $a: \widetilde{X} \rightarrow \mathbb{R}$ by the formula

$$
a(x+Z)=\left\{\begin{array}{cl}
-\inf A_{x}, & \text { if } x \in P, \\
0, & \text { if } x \in Z, \\
\inf A_{-x}, & \text { if } x \in-P .
\end{array}\right.
$$

Condition (2.3) is then straightforward. We shall prove the additivity of $a$. By the definition, if $a$ is additive on the semigroup $\widetilde{S}^{*}$, then it is additive on the whole of $\widetilde{X}$. In other words, it is enough to show that for all $x, y \in P$ we have

$$
\inf A_{x+y}=\inf A_{x}+\inf A_{y} .
$$

Fix $x, y \in P$. Let $r=\frac{m}{n}>\inf A_{x}$ and $s=\frac{m^{\prime}}{n^{\prime}}>\inf A_{y}$, where $m, m^{\prime} \in \mathbb{Z}$ and $n, n^{\prime} \in \mathbb{N}$. Then condition (2.4) implies $n x+m \xi \in P$ and $n^{\prime} y+m^{\prime} \xi \in P$. Hence

$$
n n^{\prime}(x+y)+\left(m n^{\prime}+m^{\prime} n\right) \xi=n^{\prime}(n x+m \xi)+n\left(n^{\prime} y+m^{\prime} \xi\right) \in P,
$$

which means that $r+s \in A_{x+y}$ and, in view of (2.4), $r+s \geq \inf A_{x+y}$. This proves that

$$
\inf A_{x+y} \leq \inf A_{x}+\inf A_{y} .
$$

To get the reverse inequality let $r=\frac{m}{n}<\inf A_{x}$ and $s=\frac{m^{\prime}}{n^{\prime}}<\inf A_{y}$. Then $r \notin A_{x}$, thus $n x+m \xi \notin P$ and hence, by (1.3), $k n x+k m \xi \notin P$ for every $k \in \mathbb{N}$. If we had $n x+m \xi \in Z$, then

$$
k n x+(k m+1) \xi \in Z+\xi \subset P
$$

whence

$$
A_{x} \ni \frac{k m+1}{k n} \underset{k \rightarrow \infty}{\longrightarrow} \frac{m}{n}=r ;
$$

a contradiction. Therefore, $n x+m \xi \in-P$ and, analogously, $n^{\prime} y+m^{\prime} \xi \in-P$. Hence

$$
n n^{\prime}(x+y)+\left(m n^{\prime}+m^{\prime} n\right) \xi=n^{\prime}(n x+m \xi)+n\left(n^{\prime} y+m^{\prime} \xi\right) \in-P,
$$

which means that $r+s \notin A_{x+y}$ and, in view of (2.4), $r+s \leq \inf A_{x+y}$. Consequently, equality (2.5) (and the additivity of $a$ ) has been proved. 
Define a function $g: \mathbb{R} \rightarrow \mathbb{R}$ by the formula

$$
g(x)=a(f(x)+Z) .
$$

In what follows we will show that $g$ fulfils Eq. (2.1). By (1.2), we have $g(2 x)=$ $2 g(x)$ for every $x \in \mathbb{R}$. Thus substitution $x \mapsto \frac{x+y}{2}, y \mapsto \frac{x-y}{2}$ shows that Eq. (2.1) is equivalent to the following one:

$$
g(x+y)+g(x-y)=2 \max \{g(x), g(y)\} .
$$

Putting $y=-x$ in Eq. $(*)$ and applying (1.2) we infer that $f(f(x)-$ $f(-x))=f(x)-f(-x)$, i.e. $f(x)-f(-x) \in S$ for every $x \in \mathbb{R}$. Interchanging the roles of $x$ and $-x$ we obtain $f(-x)-f(x) \in S$, therefore $f(x)-f(-x) \in Z$. This means that $g$ is an even function. Consequently, the roles of $x$ and $y$ in Eq. (2.6) are symmetric. Fix arbitrarily $x, y \in \mathbb{R}$; for the proof of equality (2.6) it is enough to distinguish the two following cases.

Case 1. If $g(x)=g(y)$, then $a(f(x)+Z)=a(f(y)+Z)$ hence $a((f(x)-f(y))+$ $Z)=0$. By virtue of (2.3), it is possible exclusively when $f(x)-f(y) \in Z$. Thus $f(x)-f(y) \in S$ and $f(y)-f(x) \in S$. Equation $(*)$ then yields

$$
2 f(x)=f(x+y)+f(x-y) \quad \text { and } \quad 2 f(y)=f(x+y)+f(y-x) .
$$

Summing up Eqs. (2.7), taking values of $a$ on both sides, and making use of the evenness of $g$ we obtain

$$
2 g(x)+2 g(y)=2 g(x+y)+g(x-y)+g(y-x)=2 g(x+y)+2 g(x-y),
$$

which is nothing else but (2.6), since $g(x)=g(y)$.

Case 2. If $g(x)>g(y)$, then $a((f(x)-f(y))+Z)>0$ and, by virtue of (2.3), $f(x)-f(y) \in P \subset S$. Equation (*) yields $2 f(x)=f(x+y)+f(x-y)$, thus $2 g(x)=g(x+y)+g(x-y)$, which gives (2.6), since $g(x)>g(y)$.

In the light of the mentioned result of Simon and Volkmann, there is an additive mapping $A: \mathbb{R} \rightarrow \mathbb{R}$ fulfilling (2.2) or, equivalently,

$$
a(f(x)+Z)=|A(x)| \text { for all } x \in \mathbb{R} .
$$

As it follows from the definition, $a$ is a monomorphism on the group $\widetilde{X}$, hence equality (2.8) gives

$$
f(x)+Z=a^{-1}(|A(x)|) \text { for all } x \in \mathbb{R} .
$$

Let $\kappa: \widetilde{X} \rightarrow X$ be an arbitrary selection (i.e. $\kappa(\omega) \in \omega$ for $\omega \in \widetilde{X}$ ). Let also $\widetilde{A}: \mathbb{R} \supset a(\widetilde{X}) \rightarrow X$ be given as $\widetilde{A}=\kappa \circ a^{-1}$. Then we may rewrite the above equality in the form

$$
f(x)=\widetilde{A}(|A(x)|)+z(x) \quad \text { for all } x \in \mathbb{R},
$$

with a suitable function $z: \mathbb{R} \rightarrow Z$. 
For an arbitrary $x \in Z$, since $f(x)=x$, we obtain $a^{-1}(|A(x)|)=0$, so it has to be $A(x)=0$. Therefore, $\left.A\right|_{Z}=0$. Since $f(f(x))=f(x)$ for $x \in \mathbb{R}$, equality (2.9) implies

$$
\widetilde{A}(|A(x)|)+z(x)=\widetilde{A}\{|A[\widetilde{A}(|A(x)|)+z(x)]|\}+z(f(x)) .
$$

By the additivity of $A$ and the fact that $A(z(x))=0$, we thus obtain

$$
\widetilde{A}(|A(x)|)-\widetilde{A}\{|A[\widetilde{A}(|A(x)|)]|\}=z(f(x))-z(x) \in Z .
$$

Hence

$$
a^{-1}(|A(x)|)=a^{-1}\{|A[\widetilde{A}(|A(x)|)]|\},
$$

therefore

$$
|A(x)|=|A[\widetilde{A}(|A(x)|)]| \quad \text { for all } x \in \mathbb{R},
$$

i.e.

$$
A(\widetilde{A}(t))= \pm t \quad \text { for all } t \in A(\mathbb{R}) .
$$

Observe that for all $x, y \in a(\tilde{X})$ we have

$$
\widetilde{A}(x+y)-\widetilde{A}(x)-\widetilde{A}(y) \in Z
$$

and since $\left.A\right|_{Z}=0$, we infer that the composition $A \circ \widetilde{A}: a(\widetilde{X}) \rightarrow X$ is additive. Consequently, Eq. (2.10) gives

$$
\left.(A \circ \widetilde{A})\right|_{A(\mathbb{R})}= \pm \mathrm{id}_{A(\mathbb{R})} .
$$

Replacing $A$ by $-A$ if needed we may assume that there is $+\operatorname{id}_{A(\mathbb{R})}$ on the righthand side. Denoting $R=\widetilde{A}(A(\mathbb{R}))$ we infer that the mappings $\left.\widetilde{A}\right|_{A(\mathbb{R})}: A(\mathbb{R}) \rightarrow$ $R$ and $\left.A\right|_{R}: R \rightarrow A(\mathbb{R})$ are bijections, and

$$
\left.\widetilde{A}\right|_{A(\mathbb{R})}=\left.A\right|_{R} ^{-1} \text {. }
$$

Because there is no algebraic structure in the set $R$ (since the selection $\kappa$ has been chosen arbitrarily), we shall now replace it by some subgroup $R^{\prime}$ of $\mathbb{R}$ such that $\left.A\right|_{R^{\prime}}: R^{\prime} \rightarrow A(\mathbb{R})$ is still a bijection. To this end it is enough to pick any Hamel basis $H$ of the linear space $\mathbb{R}$ over $\mathbb{Q}$, and define $R^{\prime}$ as the linear space spanned by a maximal set $H^{\prime} \subset H$ for which the sequence $\left(A(h): h \in H^{\prime}\right)$ is linearly independent. Then, since both $\left.A\right|_{R}: R \rightarrow A(\mathbb{R})$ and $\left.A\right|_{R^{\prime}}: R^{\prime} \rightarrow A(\mathbb{R})$ are bijections, there is a one-to-one correspondence $R \leftrightarrow R^{\prime}$ such that for all $r \leftrightarrow r^{\prime}$ we have $A(r)=A\left(r^{\prime}\right)$. This allows us to replace the selection $\kappa: \widetilde{X} \rightarrow R$ by a new selection $\kappa^{\prime}: \widetilde{X} \rightarrow R^{\prime}$ such that $\kappa(\omega) \leftrightarrow \kappa^{\prime}(\omega)$ for $\omega \in \widetilde{X}$. Defining the function $\widetilde{A}$ in the new way as $\widetilde{A}=\kappa^{\prime} \circ a^{-1}$ we obtain formula (2.9) again (with a new function $z: \mathbb{R} \rightarrow Z$ ) and all the arguments of the preceding paragraph remain valid. Therefore, with no loss of generality we may assume that equality (2.11) holds true where $R$ is a group. In particular, $\left.A\right|_{R} ^{-1}$ is additive. 
Comparing (2.11) to representation (2.9) we infer that

$$
f(x)=\left.A\right|_{R} ^{-1}(|A(x)|)+z(x) \text { for all } x \in \mathbb{R},
$$

and using Eq. $(*)$ we obtain

$$
\begin{aligned}
\left.A\right|_{R} ^{-1} & \left\{\left|A\left[\left.A\right|_{R} ^{-1}(|A(x)|)-\left.A\right|_{R} ^{-1}(|A(y)|)+z(x)-z(y)\right]\right|\right\}+z(f(x)-f(y)) \\
= & \left.A\right|_{R} ^{-1}(|A(x+y)|)+\left.A\right|_{R} ^{-1}(|A(x-y)|)-\left.A\right|_{R} ^{-1}(|A(x)|)-\left.A\right|_{R} ^{-1}(|A(y)|) \\
& +z(x+y)+z(x-y)-z(x)-z(y) .
\end{aligned}
$$

Now, applying the additivity of $A$ and $\left.A\right|_{R} ^{-1}$, identity (1.1), and the fact $A(z(x)-z(y))=0$, we conclude that

$$
z(f(x)-f(y))=z(x+y)+z(x-y)-z(x)-z(y) \text { for all } x, y \in \mathbb{R} \text {. }
$$

Putting $y=0$ in equality (2.14) we get

$$
z(f(x))=z(x) \text { for all } x \in \mathbb{R},
$$

whereas putting $x=0$ yields

$$
z(-f(x))=z(-x) \text { for all } x \in \mathbb{R} .
$$

For an arbitrary $x \in Z$, in the light of (2.12) and the fact that $\left.A\right|_{Z}=0$, we have $x=f(x)=z(x)$. Therefore

$$
\left.z\right|_{Z}=\mathrm{id}_{Z}
$$

which proves condition (iii).

Observe that $\left.z\right|_{S}$ is additive. In fact, for all $x, y \in \mathbb{R}$ we have $f(f(x)+$ $f(y))=f(x)+f(y)$ hence formula (2.12), jointly with (2.15), yields $z(f(x)+$ $f(y))=z(x)+z(y)=z(f(x))+z(f(y))$.

Assuming $|A(x)| \geq|A(y)|$ formula (2.12) gives

$$
\begin{aligned}
f(x)-f(y) & =\left.A\right|_{R} ^{-1}(|A(x)|-|A(y)|)+z(x)-z(y) \\
& =\left.A\right|_{R} ^{-1}(|A( \pm x \pm y)|)+z(x)-z(y) \\
& =f(x \pm y)-z(x \pm y)+z(x)-z(y) \in S+Z=S
\end{aligned}
$$

(with an appropriate choice of signs). Therefore, the additivity of $\left.z\right|_{S}$ and conditions (2.15), (2.17) imply $z(f(x)-f(y))=z(x)-z(y)$. By virtue of equality (2.14), $2 z(x)=z(x+y)+z(x-y)$. Representation (2.12) obviously implies that $z(2 t)=2 z(t)$ for every $t \in \mathbb{R}$, hence the foregoing equality gives

$$
z(x)=z\left(\frac{x+y}{2}\right)+z\left(\frac{x-y}{2}\right) .
$$

We are going to show that Eq. (2.18) (which holds if $|A(x)| \geq|A(y)|$ ) forces the functions $\left.z\right|_{D}$ and $\left.z\right|_{-D}$ to be additive.

Fix $t, u$ which both belong to $D$ or both to $-D$. Putting $x=t+u$ and $y=t-u$ we see that $|A(x)| \geq|A(y)|$, hence Eq. (2.18) yields $z(t+u)=$ $z(t)+z(u)$. This means that $\left.z\right|_{D}$ and $\left.z\right|_{-D}$ are additive and particularly proves condition (i). 
According to $(2.16),(2.12),(2.17)$ and the additivity of $\left.z\right|_{-D}$ we get that for every $x \in \mathbb{R}$

$$
\begin{aligned}
z(-x) & =z(-f(x))=z\left(-\left.A\right|_{R} ^{-1}(|A(x)|)-z(x)\right) \\
& =z\left(-\left.A\right|_{R} ^{-1}(|A(x)|)\right)-z(x)
\end{aligned}
$$

which proves assertion (ii). This completes the proof of the "only if" part.

For the "if" part assume $f$ is given by formula (1.6) with: an additive mapping $A: \mathbb{R} \rightarrow \mathbb{R}$, a group $R$ such that $\left.A\right|_{R}: R \rightarrow A(\mathbb{R})$ is an isomorphism, and a function $z: \mathbb{R} \rightarrow Z$, where $Z \subset \operatorname{ker} A$ is a group, which fulfill (i)-(iii). Calculation (2.13) shows that $f$ satisfies Eq. $(*)$ if and only if (2.14) holds true. In order to prove (2.14) fix $x, y \in \mathbb{R}$ and let us distinguish two possible cases.

Case 1. If $|A(x)| \geq|A(y)|$ then

$$
\begin{aligned}
f(x)-f(y) & =\left.A\right|_{R} ^{-1}(|A(x)|)-\left.A\right|_{R} ^{-1}(|A(y)|)+z(x)-z(y) \\
& =\left.A\right|_{R} ^{-1}(|A( \pm x \pm y)|)+z(x)-z(y) .
\end{aligned}
$$

Therefore, in the light of Remark 1(iv), (vi) and condition (iii),

$$
z(f(x)-f(y))=z\left(\left.A\right|_{R} ^{-1}(|A( \pm x \pm y)|)\right)+z(z(x)-z(y))=z(x)-z(y) .
$$

Thus, equality (2.14) reduces to $2 z(x)=z(x+y)+z(x-y)$ or, equivalently,

$$
z(x)=z\left(\frac{x+y}{2}\right)+z\left(\frac{x-y}{2}\right) .
$$

However, $\frac{x+y}{2}$ and $\frac{x-y}{2}$ either both belong to $D$ or both to $-D$, hence the desired equality follows from condition (i) and Remark 1(iv).

Case 2. If $|A(x)|<|A(y)|$ then

$$
\left.A\right|_{R} ^{-1}(|A(x)|)-\left.A\right|_{R} ^{-1}(|A(y)|)=\left.A\right|_{R} ^{-1}\left(-\left|A\left(\varepsilon_{1} x-\varepsilon_{2} y\right)\right|\right),
$$

where $\varepsilon_{1}, \varepsilon_{2} \in\{-1,1\}$ are such that $\varepsilon_{1} x \in D$ and $\varepsilon_{2} y \in D$. Arguing as above and using Remark 1(v) we obtain

$$
\begin{aligned}
z(f(x)-f(y)) & =z\left(\left.A\right|_{R} ^{-1}\left(-\left|A\left(\varepsilon_{1} x-\varepsilon_{2} y\right)\right|\right)\right)+z(z(x)-z(y)) \\
& =z\left(\varepsilon_{1} x-\varepsilon_{2} y\right)+z\left(-\varepsilon_{1} x+\varepsilon_{2} y\right)+z(x)-z(y) .
\end{aligned}
$$

If $\varepsilon_{1} \varepsilon_{2}=1$, then equality (2.14) reduces to $z(y-x)+2 z(x)=z(x+y)$ or, equivalently,

$$
z\left(\frac{y-x}{2}\right)+z(x)=z\left(\frac{x+y}{2}\right) .
$$

If $\varepsilon_{1} \varepsilon_{2}=-1$ then equality (2.14) reduces to $z(-x-y)+2 z(x)=z(x-y)$ or, equivalently,

$$
z\left(\frac{-x-y}{2}\right)+z(x)=z\left(\frac{x-y}{2}\right) .
$$


It is easily verified that in the first case $\frac{y-x}{2}$ and $x$ either both belong to $D$ or both to $-D$, whereas in the second case the same is true for $\frac{-x-y}{2}$ and $x$. Consequently, the desired equality follows again from condition (i) and Remark 1(iv).

\section{A new proof of the theorem of Simon and Volkmann}

We give here a new proof of the theorem of Simon and Volkmann [4] quoted above. In fact, instead of Eq. (2.1) we will be considering the following one (which was also introduced in [4]):

$$
|f(x)-f(y)|=\min \{f(x+y), f(x-y)\}
$$

under the additional assumption

$$
f(2 x)=2 f(x) \text { for all } x \in G,
$$

where $G$ is a group. As it was communicated to the author by Professor Volkmann, such a system of equations is equivalent to the equation

$$
f(x)+f(y)=\max \{f(x+y), f(x-y)\} .
$$

Indeed, if $f$ satisfies (3.3) then obviously (3.2) is valid and, moreover,

$$
\begin{aligned}
\min \{f(x+y), f(x-y)\} & =f(x+y)+f(x-y)-\max \{f(x+y), f(x-y)\} \\
& =\max \{f(2 x), f(2 y)\}-f(x)-f(y) \\
& =\max \{f(x), f(y)\}-\min \{f(x), f(y)\} \\
& =|f(x)-f(y)| \text { for all } x, y \in G .
\end{aligned}
$$

In a similar manner we may prove that (3.1) and (3.2) imply (3.3).

Observe that Eq. (3.1) immediately forces $f$ to be subadditive. Therefore, (3.2) automatically implies $f(n x)=n f(x)$ for all $x \in G$ and $n \in \mathbb{N}$. Moreover, $f^{-1}(\{0\})$ is equal to the set of periods of $f$. We will be using all these facts without explicit mentioning.

Theorem 2. Let $(G,+)$ be an Abelian group. A function $f: G \rightarrow \mathbb{R}$ is a solution of Eq. (3.1) satisfying (3.2) if and only if there exists an additive function $a: G \rightarrow \mathbb{R}$ such that $f(x)=|a(x)|$ for all $x \in G$.

Proof. The sufficiency of our assertion is easily verified, so assume that $f: G \rightarrow$ $\mathbb{R}$ is a solution of Eq. (3.1) such that $f(n x)=n f(x)$ for all $x \in G$ and $n \in \mathbb{N}$. For transparency we divide the proof into several parts. In parts I-IV we additionally assume that $G$ is an Abelian free group. In the last part we will show how to reduce the general case to that special one.

I. Let $\left\{h_{t}\right\}_{t \in T}$ be any basis of the free group $G$ and denote

$$
v_{t}=f\left(h_{t}\right) \quad \text { for } t \in T \text {. }
$$


Let $\mathcal{C}$ be the family consisting of all sequences with the domain $T$ and taking values in the set $\{-1,1\}$ and let

$$
U=\left\{t \in T: v_{t} \neq 0\right\} .
$$

We introduce an equivalence relation $\sim$ in $\mathcal{C}$ given by the formula

$$
\left(\delta_{t}\right)_{t \in T} \sim\left(\varepsilon_{t}\right)_{t \in T} \Leftrightarrow\left\{\begin{array}{l}
\delta_{t}=\varepsilon_{t} \quad \text { for all } t \in U, \text { or } \\
\text { the sequences }\left.\delta\right|_{U} \text { and }\left.\varepsilon\right|_{U} \text { are constant. }
\end{array}\right.
$$

The last condition is equivalent to the following one: if $a_{\delta}, a_{\varepsilon}$ are additive functions satisfying $a_{\delta}\left(h_{t}\right)=\delta_{t} v_{t}$ and $a_{\varepsilon}\left(h_{t}\right)=\varepsilon_{t} v_{t}$ for $t \in T$, then $\left|a_{\delta}\right|=\left|a_{\varepsilon}\right|$.

Take arbitrarily $t, u \in U, t \neq u$ (if any such pair $(t, u)$ exists). Then

$$
f\left(h_{t}+h_{u}\right) \neq f\left(h_{t}-h_{u}\right) .
$$

Indeed, suppose that it is not the case. Putting $x=h_{t}+h_{u}, y=h_{t}-h_{u}$ in Eq. (3.1) we then obtain either $v_{t}=0$ or $v_{u}=0$, which is a contradiction. Consequently, exactly one of the two values $f\left(h_{t}+h_{u}\right), f\left(h_{t}-h_{u}\right)$ equals $\left|v_{t}-v_{u}\right|$. In the case where the first one does, we define $\sigma_{t, u}=-1$, if the latter one does, we define $\sigma_{t, u}=1$. There exists exactly one (up to the equivalence $\sim$ ) sequence $\left(\varepsilon_{t}\right)_{t \in T} \in \mathcal{C}$ fulfilling

$$
\varepsilon_{t} \varepsilon_{u}=\sigma_{t, u} \text { for all } t, u \in U, t \neq u \text {. }
$$

To see this it is enough to check the following transitivity condition: if $t, u$, $w \in U$ are pairwise different and $\sigma_{t, u}=1=\sigma_{u, w}$, then $\sigma_{t, w}=1$. Indeed, by the definition of $\sigma_{t, u}$ and $\sigma_{u, w}$, we have

$$
f\left(h_{t}-h_{u}\right)=\left|v_{t}-v_{u}\right|
$$

and

$$
f\left(h_{u}-h_{w}\right)=\left|v_{u}-v_{w}\right| .
$$

Observe that independently of the values $\left|v_{t}-v_{u}\right|$ and $\left|v_{u}-v_{w}\right|$ we can either add or subtract these two equations reducing the term $v_{u}$ on the right-hand side. As a result we get one of the two equalities:

$$
f\left(h_{t}-h_{u}\right)+f\left(h_{u}-h_{w}\right)=\left|v_{t}-v_{w}\right|,
$$

or

$$
\left|f\left(h_{t}-h_{u}\right)-f\left(h_{u}-h_{w}\right)\right|=\left|v_{t}-v_{w}\right| .
$$

Formula (3.4), jointly with the subadditivity of $f$, yields

$$
f\left(h_{t}-h_{w}\right) \leq\left|f\left(h_{t}\right)-f\left(h_{w}\right)\right| .
$$

However, Eq. (3.1) implies then that the above inequality is in fact an equality, i.e. $\sigma_{t, w}=1$. In the case where formula (3.5) is valid, suppose $\sigma_{t, w}=-1$, which means that $f\left(h_{t}+h_{w}\right)=\left|v_{t}-v_{w}\right|$ and (3.5) yields

$$
\left|f\left(h_{t}-h_{u}\right)-f\left(h_{u}-h_{w}\right)\right|=f\left(h_{t}+h_{w}\right) .
$$


Therefore, there are two possibilities:

$$
f\left(h_{t}+h_{w}\right)= \pm\left(f\left(h_{t}-h_{u}\right)-f\left(h_{u}-h_{w}\right)\right) .
$$

The first one implies

$$
f\left(h_{t}-h_{u}\right)=f\left(h_{t}+h_{w}\right)+f\left(h_{u}-h_{w}\right) \geq f\left(h_{t}+h_{u}\right)
$$

and hence, by Eq. (3.1),

$$
f\left(h_{t}+h_{u}\right)=\left|v_{t}-v_{u}\right|,
$$

which contradicts $\sigma_{t, u}=1$. The latter possibility similarly leads to contradiction with $\sigma_{u, w}=1$.

We have assigned to our solution $f$ the two sequences: $\left(v_{t}\right)_{t \in T}$ and $\left(\varepsilon_{t}\right)_{t \in T}$. Our present aim is to show that these two sequences completely determine all the values of the function $f$.

II. In the first step we will show that the values of $f$ are determined for all arguments being linear combinations of exactly two elements from the basis $H$. Fix $t, u \in T, t \neq u$. We may assume that $t, u \in U$; otherwise at least one of the numbers $h_{t}, h_{u}$ is a period of $f$, which obviously implies that all values $f\left(m h_{t}+n h_{u}\right)$ are determined for $m, n \in \mathbb{Z}$. Denote by $\mathcal{S}$ the family of all 2-homogeneous solutions of Eq. (3.1) to which we have assigned the same sequences $\left(v_{t}\right)_{t \in T},\left(\varepsilon_{t}\right)_{t \in T}$ as those assigned to the solution $f$, and let

$$
\begin{aligned}
\mathcal{N}_{t, u}= & \{(m, n) \in \mathbb{Z} \times \mathbb{Z}: \text { for every } g \in \mathcal{S} \text { we have } \\
& \left.f\left(m h_{t}+n h_{u}\right)=g\left(m h_{t}+n h_{u}\right)\right\} .
\end{aligned}
$$

We shall prove that

$$
\mathbb{Z} \times \mathbb{Z}=\mathcal{N}_{t, u}
$$

First, observe that both pairs $(1,1),(1,-1)$ belong to $\mathcal{N}_{t, u}$. Indeed, in the case where $\sigma_{t, u}=1$ we have

$$
f\left(h_{t}-h_{u}\right)=\left|v_{t}-v_{u}\right| .
$$

If, moreover, $v_{t} \geq v_{u}$ then

$$
f\left(2 h_{t}+h_{u}\right) \geq 2 v_{t}-v_{u} \geq v_{u},
$$

thus the substitution $x \mapsto h_{t}+h_{u}, y \mapsto h_{t}$ into Eq. (3.1) yields

$$
\left|f\left(h_{t}+h_{u}\right)-v_{t}\right|=v_{u},
$$

which implies either

$$
f\left(h_{t}+h_{u}\right)=v_{t}+v_{u}
$$

or

$$
f\left(h_{t}+h_{u}\right)=v_{t}-v_{u}
$$


However, the last equality and equality (3.7) give $f\left(h_{t}+h_{u}\right)=f\left(h_{t}-h_{u}\right)$, contrary to the assumption $t, u \in U$. Consequently, Eqs. (3.7) and (3.8) determine the values $f\left(h_{t}-h_{u}\right)$ and $f\left(h_{t}+h_{u}\right)$ uniquely in the case $v_{t} \geq v_{u}$. The opposite case may be treated similarly: we have

$$
f\left(h_{t}+h_{u}\right)=\left|v_{t}-v_{u}\right|
$$

and

$$
f\left(h_{t}-2 h_{u}\right) \geq 2 v_{u}-v_{t} \geq v_{t},
$$

which, in view of the substitution $x \mapsto h_{t}-h_{u}, y \mapsto h_{u}$, yields

$$
\left|f\left(h_{t}-h_{u}\right)-v_{u}\right|=v_{t} .
$$

Like in the previous case we exclude one of the possibilities and we obtain

$$
f\left(h_{t}-h_{u}\right)=v_{u}-v_{t} .
$$

Analogous arguments work in the case where $\sigma_{t, u}=-1$. Consequently,

$$
(1,1),(1,-1) \in \mathcal{N}_{t, u} \text {. }
$$

Before proceeding to the next part of the proof of our claim (3.6), we shall distinguish two possibilities: $v_{t} \geq v_{u}$ and $v_{u} \geq v_{t}$.

Case $1\left(v_{t} \geq v_{u}\right)$. We are going to prove that

$$
\mathcal{N}^{+}:=\{(m, n) \in \mathbb{Z} \times \mathbb{Z}:|m| \geq|n|\} \subset \mathcal{N}_{t, u}
$$

using an induction on $|m|-|n|$. By virtue of (3.10) and the homogeneity of $f$, every pair $(m, n) \in \mathcal{N}^{+}$with $|m|=|n|$ belongs to $\mathcal{N}_{t, u}$. Now, fix $r \in \mathbb{N}$ and $(m, n) \in \mathcal{N}^{+}$with $|m|-|n|=r$, and assume that

$$
\{(m, n) \in \mathbb{Z} \times \mathbb{Z}: 0 \leq|m|-|n|<r\} \subset \mathcal{N}_{t, u} .
$$

With no loss of generality we may suppose that $m \in \mathbb{N}, m \geq 2$.

First, consider the case where $n \in \mathbb{N}$ (and hence $1 \leq n<m=n+r$ ). For arbitrary $k, l \in \mathbb{Z}, k>l$, putting $x \mapsto k h_{t}+l h_{u}, y \mapsto h_{t}$ and $x \mapsto k h_{t}+l h_{u}$, $y \mapsto h_{u}$ in Eq. (3.1), we obtain

$$
\left|f\left(k h_{t}+l h_{u}\right)-v_{t}\right|=\min \left\{f\left((k+1) h_{t}+l h_{u}\right), f\left((k-1) h_{t}+l h_{u}\right)\right\}
$$

and

$$
\left|f\left(k h_{t}+l h_{u}\right)-v_{u}\right|=\min \left\{f\left(k h_{t}+(l+1) h_{u}\right), f\left(k h_{t}+(l-1) h_{u}\right)\right\} ;
$$

moreover,

$$
f\left(k h_{t}+l h_{u}\right) \geq k v_{t}-l h_{u} \geq \max \left\{v_{t}, v_{u}\right\} .
$$

Hence

$$
\bigwedge_{\substack{k, l \in \mathbb{Z} \\
k>l}}\left\{\begin{array}{l}
(k+1, l),(k-1, l) \in \mathcal{N}_{t, u}, \text { or } \\
(k, l+1),(k, l-1) \in \mathcal{N}_{t, u}
\end{array} \Rightarrow(k, l) \in \mathcal{N}_{t, u} .\right.
$$

Applying this implication, the inductive hypothesis and 2-homogeneity we get: 
(a) if $m, n$ are even then

$$
\left(\frac{m}{2}, \frac{n}{2}\right) \in \mathcal{N}_{t, u} \Rightarrow(m, n) \in \mathcal{N}_{t, u}
$$

(b) if $m$ is even, $n$ is odd then

$$
\begin{aligned}
& \left(\frac{m}{2}, \frac{n-1}{2}\right) \in \mathcal{N}_{t, u} \Rightarrow(m, n-1) \in \mathcal{N}_{t, u}, \\
& (m, n-1),(m, n+1) \in \mathcal{N}_{t, u} \Rightarrow(m, n) \in \mathcal{N}_{t, u} ;
\end{aligned}
$$

(c) if $m$ is odd, $n$ is even then

$$
\begin{aligned}
& \left(\frac{m+1}{2}, \frac{n}{2}\right) \in \mathcal{N}_{t, u} \Rightarrow(m+1, n) \in \mathcal{N}_{t, u}, \\
& (m-1, n),(m+1, n) \in \mathcal{N}_{t, u} \Rightarrow(m, n) \in \mathcal{N}_{t, u} ;
\end{aligned}
$$

(d) if $m, n$ are odd then

$$
\begin{aligned}
& \left(\frac{m+1}{2}, \frac{n+1}{2}\right),\left(\frac{m+1}{2}, \frac{n+1}{2}\right) \in \mathcal{N}_{t, u} \\
& \quad \Rightarrow(m+1, n-1),(m+1, n+1) \in \mathcal{N}_{t, u} \\
& \quad \Rightarrow(m+1, n) \in \mathcal{N}_{t, u}, \\
& (m-1, n),(m+1, n) \in \mathcal{N}_{t, u} \Rightarrow(m, n) \in \mathcal{N}_{t, u} .
\end{aligned}
$$

The case where $n<0$ may be treated as follows. We put $x \mapsto m h_{t}+n h_{u}$, $y \mapsto m h_{t}-n h_{u}$ and $x \mapsto m h_{t}, y \mapsto n h_{u}$ in Eq. (3.1) obtaining

$$
\left|f\left(m h_{t}+n h_{u}\right)-f\left(m h_{t}-n h_{u}\right)\right|=\min \left\{2 m v_{t}, 2 n v_{u}\right\}
$$

and

$$
\min \left\{f\left(m h_{t}+n h_{u}\right), f\left(m h_{t}-n h_{u}\right)\right\}=\left|m v_{t}-n v_{u}\right| .
$$

These two equations determine the value $f\left(m h_{t}+n h_{u}\right)$, since we already know the values: $f\left(m h_{t}-n h_{u}\right), v_{t}, v_{u}$. This completes the proof of (3.11).

It remains to show that

$$
\mathcal{N}^{-}:=\{(m, n) \in \mathbb{Z} \times \mathbb{Z}:|m| \leq|n|\} \subset \mathcal{N}_{t, u} .
$$

Fix any pair $(m, n) \in \mathcal{N}^{-}$; we may assume that $m \neq 0$. Choose $k \in \mathbb{N}$ such that $(k-1)|m|>|n|$ and substitute $x \mapsto m h_{t}+n h_{u}, y \mapsto k m h_{t}$ in Eq. (3.1). We get

$$
\left|f\left(m h_{t}+n h_{u}\right)-k\right| m\left|v_{t}\right|=\min \left\{f\left((1+k) m h_{t}+n h_{u}\right), f\left((1-k) m h_{t}+n h_{u}\right)\right\} .
$$

The two values on the right-hand side are already determined. Moreover,

$$
f\left(m h_{t}+n h_{u}\right) \leq|m| v_{t}+|n| v_{u} \leq k|m| v_{t}
$$

and, consequently, Eq. (3.13) determines the value $f\left(m h_{t}+n h_{u}\right)$, i.e. $(m, n) \in$ $\mathcal{N}_{t, u}$. 
Case $2\left(v_{u} \geq v_{t}\right)$. We argue analogously to the previous case; we start with showing (3.12) and then deduce (3.11) from (3.12).

Summarizing this part of the proof we may write

$$
\mathbb{Z} \times \mathbb{Z}=\mathcal{N}^{+} \cup \mathcal{N}^{-} \subset \mathcal{N}_{t, u},
$$

which gives (3.6).

III. Now we will show that the value $f(x+y+z)$ is determined provided we know the values of $f$ on every sum of not more that two terms from $\{x, y, z\}$. In the light of the preceding part of the proof, it would imply that all the values of $f$ are completely determined by the sequences $\left(v_{t}\right)_{t \in T}$ and $\left(\varepsilon_{t}\right)_{t \in T}$.

Applying Eq. (3.1) repeatedly we get

$$
\begin{aligned}
& \min \{f(x+y+z), f(x+y-z)\}=|f(x+y)-f(z)|, \\
& \min \{f(x+y+z), f(x-y+z)\}=|f(x+z)-f(y)|, \\
& \min \{f(x+y+z), f(x-y-z)\}=|f(y+z)-f(x)| .
\end{aligned}
$$

Let $a \geq b \geq c \geq d$ be all the values: $f(x+y+z), f(x+y-z), f(x-y+z)$, $f(x-y-z)$. We may assume that they are pairwise different, since otherwise one of the sums of not more than two terms from $\{x, y, z\}$ would be a period of $f$, which automatically determines $f(x+y+z)$.

If the three expressions on the right-hand sides of Eqs. (3.14) occur not to be pairwise different, then the repeating one has to be equal to $f(x+y+z)$. In the opposite case we have

$$
f(x+y+z) \in\{a, b\}
$$

and at least one of the numbers $c, d$ appears in one of the right-hand sides. Then, taking the equation with the least right-hand side, we get

$$
\min \{f(x+y+z), f(x \pm y \pm z)\} \in\{c, d\},
$$

which determines the value $e:=f(x \pm y \pm z)$, where the sequence of signs is one of: $(+,-),(-,+),(-,-)$. Applying Eq. (3.1) once more we obtain

$$
|f(x+y+z)-e|=\min \left\{2 f\left(x^{\prime}\right), 2 f\left(x^{\prime \prime}\right)\right\},
$$

where each of $x^{\prime}, x^{\prime \prime}$ is a sum of not more than two terms from $\{x, y, z\}$. However,

$$
\{a, b\} \ni f(x+y+z) \geq e \in\{c, d\},
$$

thus formula (3.15) determines $f(x+y+z)$.

IV. Let $a: G \rightarrow \mathbb{R}$ be the additive function satisfying $a\left(h_{t}\right)=\varepsilon_{t} v_{t}$ for $t \in T$. Then $|a|$ is a solution of our equation to which we assign the same sequence $\left(v_{t}\right)_{t \in T},\left(\varepsilon_{t}\right)_{t \in T}$ as those assigned to $f$. By virtue of parts II and III, it has to be $f=|a|$.

V. Now, assume that $G$ is an arbitrary Abelian group (not necessarily free). Then $G$ is an image of a certain Abelian free group $H$ through a homomorphism $\varphi: H \rightarrow G$. By the isomorphism theorem, there is an isomorphism 
$\Phi: H / \operatorname{ker} \varphi \rightarrow G$, such that $\Phi \circ \kappa=\varphi$, where $\kappa: H \rightarrow H / \operatorname{ker} \varphi$ is the canonical projection. Let $\tilde{f}: H \rightarrow \mathbb{R}$ be given as $\widetilde{f}=f \circ \varphi$. The function $\tilde{f}$, being a 2-homogenous solution of the equation considered, which acts on an Abelian free group, admits the representation $\widetilde{f}=|\widetilde{a}|$ for some additive function $\widetilde{a}: H \rightarrow \mathbb{R}$. Define a mapping $a: G \rightarrow \mathbb{R}$ by the formula

$$
a(\Phi(x+\operatorname{ker} \varphi))=\widetilde{a}(x) .
$$

It is easy to see that $\widetilde{a}(x)$ does not depend on the specific member of the coset $x+\operatorname{ker} \varphi$. Moreover, $a$ is additive and for every $y \in G, y=\Phi(x+\operatorname{ker} \varphi)$ for some $x \in H$, we have

$$
f(y)=f(\Phi \circ \kappa)(x)=(f \circ \varphi)(x)=\widetilde{f}(x)=|\widetilde{a}(x)|=|a(y)|,
$$

which completes the proof.

\section{Concluding remarks}

Now, we are going to deduce some corollaries from our main result. The first one requires the following theorem due to Maksa and Rätz [2] and extended by Sablik (cf. [3, Proposition 3.2]). Namely, if $G$ is a group and $a, b: G \rightarrow \mathbb{R}$ are additive mappings satisfying $a^{-1}((0, \infty))=b^{-1}((0, \infty))$, then there is a positive number $\gamma$ such that $a=\gamma b$.

Corollary 1. Assume $f: \mathbb{R} \rightarrow[0, \infty)$ is a function satisfying $\left(\mathrm{H}_{1}\right)$. Then $f$ is a solution of Eq. (*) if and only if there exists an additive function $A: \mathbb{R} \rightarrow \mathbb{R}$ such that $A \circ A=A$ and

$$
f(x)=|A(x)| \quad \text { for all } x \in \mathbb{R} .
$$

Proof. It is easily verified that every function of the form (4.1) fulfills $(*)$. Assuming now that $f$ is a non-negative solution of $(*)$ fulfilling $\left(\mathrm{H}_{1}\right)$ we infer, in view of Remark 2, that $\left(\mathrm{H}_{2}\right)$ holds true. Thus, Theorem 1 gives (1.6) with appropriate functions $A$ and $z: \mathbb{R} \rightarrow Z$. However, since $S \subset[0, \infty)$, condition (1.3) implies $Z=\{0\}$ and consequently $z$ vanishes. Therefore

$$
f(x)=A_{R}^{-1}(|A(x)|) \text { for all } x \in \mathbb{R} .
$$

Now, consider the group $A(\mathbb{R})$ and the function $\left.A\right|_{R} ^{-1}: A(\mathbb{R}) \rightarrow \mathbb{R}$. This is an additive mapping which takes positive values exactly on the set $A(\mathbb{R}) \cap(0, \infty)$. The same is trivially true for the identity function on $A(\mathbb{R})$. Thus, by the above mentioned result of Maksa, Rätz and Sablik, there is a positive number $\gamma$ such that $\left.A\right|_{R} ^{-1}(A(x))=\gamma A(x)$ for all $x \in \mathbb{R}$. Hence, obviously $\left.A\right|_{R} ^{-1}(|A(x)|)=$ $\gamma|A(x)|$ and, by formula (4.2), $f(x)=\lambda|A(x)|$ for all $x \in \mathbb{R}$. Putting this into Eq. (*) immediately gives $\lambda=1$, thus (4.1) has been proved. 
Corollary 2. Assume $f: \mathbb{R} \rightarrow(-\infty, 0]$ is a function satisfying $\left(\mathrm{H}_{1}\right)$. Then $f$ is a solution of Eq. $(*)$ if and only if there exists an additive function $A: \mathbb{R} \rightarrow \mathbb{R}$, such that $A \circ A=A$ and

$$
f(x)=-|A(x)| \text { for all } x \in \mathbb{R} .
$$

Proof. It is enough to consider, instead of $f(x)$, the function $-f(-x)$ and to apply Corollary 1.

Corollary 3. Assume $f: \mathbb{R} \rightarrow \mathbb{R}$ is an even function satisfying $\left(\mathrm{H}_{1}\right)$ and $\left(\mathrm{H}_{2}\right)$. Then $f$ is a solution of Eq. (*) if and only if there exist: an additive function $A: \mathbb{R} \rightarrow \mathbb{R}$ and a subgroup $R$ of $\mathbb{R}$, such that $\left.A\right|_{R}: R \rightarrow A(\mathbb{R})$ is an isomorphism, such that

$$
f(x)=\left.A\right|_{R} ^{-1}(|A(x)|) \quad \text { for all } x \in \mathbb{R} .
$$

Proof. If $f$ is an even function, then (1.6) implies that $z$ is even as well. Making use of condition (iii) for every $x \in Z$ we obtain $x=z(x)=z(-x)=-x$, which proves that $Z=\{0\}$. Consequently, $z$ vanishes and formula (1.6) reduces to (4.3).

Even under additional assumptions $\left(\mathrm{H}_{1}\right)$ and $\left(\mathrm{H}_{2}\right)$ the description of solutions of Eq. $(*)$ is rather complicated and we have a variety of them. What is more, the general situation is even worse (or better - depends on the point of view). Namely, if $T \subset \mathbb{R}$ is an arbitrary semigroup satisfying:

$\left(\mathrm{T}_{1}\right) T \cup(-T)=T-T$,

$\left(\mathrm{T}_{2}\right) T \cap(-T)=\{0\}$,

then the function $\chi_{T}: T \cup(-T) \rightarrow T \cup(-T)$ defined by

$$
\chi_{T}(x)= \begin{cases}x, & \text { if } x \in T, \\ -x, & \text { if } x \in-T,\end{cases}
$$

satisfies the equation considered, i.e.

$$
\begin{aligned}
& \chi_{T}\left(\chi_{T}(x)-\chi_{T}(y)\right)=\chi_{T}(x+y)+\chi_{T}(x-y)-\chi_{T}(x)-\chi_{T}(y) \\
& \quad \text { for all } x, y \in T \cup(-T) .
\end{aligned}
$$

In particular, taking a Hamel basis $\left\{h_{\alpha}\right\}_{\alpha \in A}$ with a well-ordered set $(A, \leq)$, we may define

$$
T=\left\{x \in \mathbb{R}: x=\sum_{i=1}^{n} \lambda_{i} h_{\alpha_{i}}, \lambda_{i} \in \mathbb{Q}, \alpha_{1} \leq \cdots \leq \alpha_{n} \text { and } \lambda_{n}>0\right\} .
$$

Then $\chi_{T}$ yields a solution of the above equation, whereas condition $\left(\mathrm{H}_{2}\right)$ does not hold true.

We can make the above example even a bit more complicated by choosing an arbitrary additive function $A: \mathbb{R} \rightarrow \mathbb{R}$ and a subgroup $R$ of $\mathbb{R}$ such that $\left.A\right|_{R}: R \rightarrow A(\mathbb{R})$ is an isomorphism. If a semigroup $T \subset \mathbb{R}$ satisfies conditions 
$\left(\mathrm{T}_{1}\right),\left(\mathrm{T}_{2}\right)$ and $A(\mathbb{R}) \subset T \cup(-T)$, then the composition $f=\left.A\right|_{R} ^{-1} \circ \chi_{T} \circ A$ fulfills Eq. (*).

Taught by the examples above, the author does not attempt to give any conjecture about the general form of solutions $f: \mathbb{R} \rightarrow \mathbb{R}$ of Eq. (*) with no additional assumptions. Nevertheless, one conjecture seems to be reasonable. Namely, for every solution which is known to the author, assumption $\left(\mathrm{H}_{1}\right)$ occurs to be true. It seems probable that this is a general rule.

Open Access. This article is distributed under the terms of the Creative Commons Attribution Noncommercial License which permits any noncommercial use, distribution, and reproduction in any medium, provided the original author(s) and source are credited.

\section{References}

[1] Fechner, W.: On a composite functional equation on Abelian groups. Aequ. Math. 78, 185-193 (2009)

[2] Maksa, Gy., Rätz, J.: Remark 5. In: Proceedings of the 19th ISFE, Nantes, La Turballe, France, 1981, Centre for Information Theory, University of Waterloo (1981)

[3] Sablik, M.: Basic Sets for Functional Equations. Uniwersytetu Ślasskiego, Katowice (1996)

[4] Simon, A., Volkmann, P.: Caractérisation du module d'une fonction additive à l'aide d'une équation fonctionnelle. Aequ. Math. 47, 60-68 (1994)

[5] Tarski, A.: Problem no. 83. Parameter 1. (6), 231 (1930); Solution: Młody Matematyk 1. (1), 90 (1931) (in Polish)

Tomasz Kochanek

Institute of Mathematics

University of Silesia

Bankowa 14

40-007 Katowice

Poland

e-mail: tkochanek@math.us.edu.pl

Received: June 27, 2009

Revised: January 23, 2010 